\title{
Höhere Rationalität durch Bewertung?
}

\author{
Am Beispiel der produktbezogenen Ökobilanz behandelt der folgende Beitrag \\ vor allem die Frage, wie vielfältige, komplexe Informationen bewältigt wer- \\ den können. Außerdem wird erörtert, welchen Beitrag Bewertungen hierzu für \\ verschiedene Akteure liefern können.
}

\section{Von Katbrin Ankele und Frieder Rubik \\ Die Bewerfung im Rahmen der Ökobilanz}

ewertung kann beschrieben werden als „die Verknüpfung der zugänglichen Informationen eines Sachverhaltes mit dem persönlichen Wertesystem zu einem Urteil über den entsprechenden Sachverhalt" (Giegrich u.a. 1995, S. 5). Sachinformationen werden also mit subjektiven Werten verbunden. Diesen Vorgang haben alle Bewertungen gemeinsam. Bewertung hilft, die Informationsvielfalt $\mathrm{zu}$ strukturieren und deren Komplexität zu verringern, indem Informationen verdichtet werden.

Was heißt in diesem Zusammenhang höhere Rationalität? Als rational werden in der Regel Handlungen und Entscheidungen bezeichnet, die vom Verstand, von der Vernunft, bestimmt und geleitet werden. Wie paßt diese Anforderung mit der vorab formulierten Definition von Bewertung zusammen? Dieser Frage wollen wir nachgehen und klären, was eine Bewertungsmethode leisten kann. Wir beziehen uns dabei auf die Bewertung im Rahmen von produktbezogenen Ökobilanzen.

Ökobilanzen haben seit Anfang der 90er Jahre als ökologisches Informationsinstrument zur Erfassung und Bewertung der entlang eines Produktlebenszyklusses auftretenden Umweltwirkungen, das heißt von der Rohstoffgewinnung über die Produktion und den Gebrauch bis zur Entsorgung einschließlich aller Transporte, an Bedeutung gewonnen (vgl. Rubik/Teichert 1997). Sie sind ein Instrument moderner Umweltpolitik und Umweltmanagements. Aufbauend auf der Erhebung der Stoff- und Energieverbräuche sowie der Emissionen und Abfälle in einer "Sachbilanz“, findet eine ökologische Bewertung der Daten der Sachbilanz in der „Wirkungsabschätzung“" und der „Auswertung“ statt.
Ökobilanzen wurden entwickelt, um Produkte und ihre Eigenschaften umfassender zu beurteilen. Standen früher technisch-funktionale sowie ökonomische Eigenschaften im Mittelpunkt, so rückte einhergehend mit der allgemeinen umweltpolitischen Sensibilisierung auch die ökologische Dimension in den Vordergrund. Ökobilanzen sind mittlerweile ein sehr häufig verwendetes Informationsinstrument. Mit ihrer Verwendung wird die Hoffnung eines ,aufgeklärteren“ Produktions- und Nutzungsverhaltens von Produkten verbunden. Ökobilanzen sollen somit einen Beitrag zur Verringerung der Umweltbelastungen leisten und in Richtung einer nachhaltigen Entwicklung führen.

Bereits in der Vergangenheit mußten bei produktbezogenen Entscheidungen mehrere Dimensionen berücksichtigt werden. Durch Ökobilanzen wird nun eine weitere Dimension die der Ökologie - in Entscheidungs- und Abwägungsprozesse eingebracht. Die Mehrdimensionalität der Betrachtung wird erweitert, da keine der zuvor im Entscheidungskranz vorhandenen Dimensionen „ausgemustert“. wird. Wie kann mit dieser Mehrdimensionalität im Rahmen einer Ökobilanz umgegangen werden? Zwei Ansätze sind hier zu nennen, wobei wir uns im weiteren auf den zweiten Ansatz beschränken wollen:

(1) Zum einen werden die ökologischen Informationen, die in der Sachbilanz erfaßt wurden, zusammengefaßt und bewertet.

(2) Zum anderen werden aus dem Untersuchungsrahmen einer Ökobilanz weitere Dimensionen per definitionem ausgeklammert: Eine Ökobilanz wird begrenzt auf eine ökologische Bilanz von Produkten. Andere entscheidungsrelevante Informationen werden dagegen nicht mit der Ökobilanz verbunden, sondern sind auf andere Weise zu berücksichtigen.

Die in einer Sachbilanz erhobenen Informationen sind in den letzten Jahren sprunghaft angestiegen. Dies hängt sicherlich damit zusammen, $\mathrm{da}$ eine große Unsicherheit darüber besteht, welches die wesentlichen Parameter sind, die in Sachbilanzen erhoben werden sollen. Es gibt keinen wissenschaftlichen und umweltpolitischen Konsens über eine Liste von einigen wenigen Leitparametern. In dieser Unsicherheitslage werden lieber zu viele als zu wenige Parameter erhoben. Dies wird erleichtert durch die zunehmende Professionalisierung des Umweltmanagements und die Unterstützung durch EDV. Mittlerweile gibt es viele Ökobilanzen, deren Sachbilanz mehr als 100 verschiedene Parameter enthält.

Wie sollen derartig komplexe Informationsberge bewältigt werden? Betriebliche Entscheidungsträger haben im Regelfall den Wunsch, die Vielzahl der Informationen zu verringern, indem diese verdichtet und bewertet werden. Nur auf diese Weise kann die ökologische Dimension überhaupt in den betrieblichen Entscheidungsprozeß eingebunden werden.

Verbraucher/innen leiden unter der ,information overload", der Informationsüberlastung. Empirische Untersuchungen (vgl. z. B. Rubik/Weskamp 1996) weisen eindeutig darauf hin, daß diese einen Bedarf nach knappen und rasch nachvollziehbaren Produktinformationen haben, um diese überhaupt in die Kaufentscheidungen einzubeziehen. Die Rolle der Informationsverdichtung und -aufbereitung wird nun vielfach der Bewertungsmethode zugeschrieben. Auch die Umweltpolitik hat eine gewisse ,Sehnsucht" nach einfachen Ergebnissen und nicht komplexen Antworten. Komplizierte Abwägungsprozesse innerhalb und zwischen sehr verschiedenen Aspekten sind kaum noch vermittelbar. Hier steht die fachwissenschaftliche Politikberatung (u. a. auch des UBA) vor einer großen Herausforderung. Die verschiedenen Nutzer und Entscheidungsträger haben somit einen Bedarf an verdichteten Informationen, die die Komplexität einer Ökobilanz reduzieren und der Informationsüberlastung gegensteuern.

\section{Zwei Schritte}

In der aktuellen Ökobilanzdiskussion wird zwischen zwei bewertungsrelevanten Schritten differenziert (vgl. Ankele/Rubik 1997): 
(1) Zum einen ist dies die mit „Wirkungsabschätzung" bezeichnete Zuordnung von Stoffflüssen $\mathrm{zu}$ den Umweltwirkungen. In diesem Schritt wird die Vielzahl der Sachbilanzdaten im Hinblick auf ihre ökologische Relevanz vorstrukturiert (klassifiziert) und zusammengefaßt (charakterisiert), um die anschließende Bewertung vorzubereiten. Das Spezifische und Neue an der Wirkungsabschätzung ist, daß sich die Strukturierung an den potentiellen Umweltwirkungen orientiert, die durch die jeweiligen Ressourcenentnahmen, Emissionen, etc. hervorgerufen werden: Die im Untersuchungssystem auftretenden Emissionen werden mit derzeit bekannten und nachvollziehbaren Umweltwirkungen wie Treibhauseffekt, Eutrophierung etc. in Zusammenhang gebracht.

Allerdings können nie alle potentiell auftretenden Umweltwirkungen bearbeitet werden, eine Beschränkung auf die wichtigsten ist nötig. Eine methodische Konvention, die derzeit in den internationalen Normungsgremien erarbeitet wird, soll daher die Wirkungskategorien festschreiben, die in jeder Ökobilanz bearbeitet werden sollen. Ferner muß davon ausgegangen werden, daß Zeitverzögerungen zwischen der Diskussion einer Umweltbelastung in Fachkreisen und der Anerkennung in politischen Zusammenhängen nicht zu vermeiden sind.

(2) Zum anderen ist dies die Verknüpfung von Sachinformationen (der Sachbilanz und der Wirkungsabschätzung) mit Wertehaltungen.

Diese Verknüpfung ist bisher noch kaum vereinheitlicht, sondern geschieht auf verschiedene Weisen:

- Eine Möglichkeit ist die Festlegung bestimmter Bewertungskriterien, wie beispielsweise die ökologische Bedeutung. Darunter ist zu verstehen, daß die in der Sachbilanz und/oder der Wirkungsabschätzung ermittelten Umweltwirkungen im Hinblick auf verschiedene Kriterien hierarchisiert werden. Weitere Bewertungskriterien sind beispielsweise ökologisches Gefährdungspotential, zeitliche Reichweite, Reversibilität/Irreversibilität, räumliche Reichweite, Verhältnis der Ist-Situation zu Qualitätszielen (vgl. Schmitz 1995, Ankele/Rubik 1997). Diese Zuordnung ist von einer anderen Qualität als die in der Wirkungsabschätzung und stärker durch die subjektive Einschätzung der Bewertenden gekennzeichnet. Das Resultat kann eine grobe ordinale Einstufung sein.

- Eine andere Möglichkeit ist die - eventuell argumentativ gestïtzte - Vergabe von Gewich-

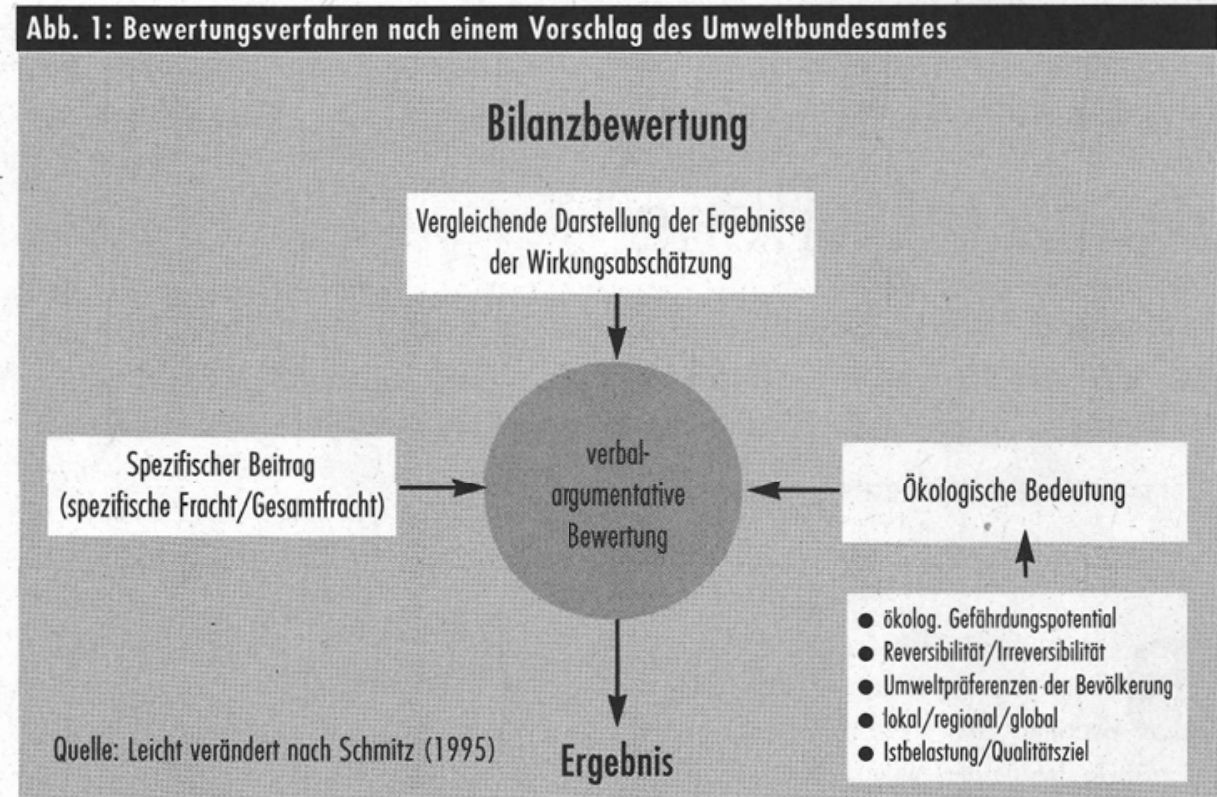

tungen in Anlehnung an die Nutzwertanalyse. Die abschließende Beurteilung und damit Beantwortung der zu Beginn der Ökobilanz formulierten Fragen wird durch die Zusammenführung dieser beiden Bestandteile zu einem Gesamturteil realisiert. Abbildung 1 zeigt hierzu exemplarisch das Bewertungsverfahren des Umweltbundesamtes, das in der Ökobilanz für Getränkeverpackungen entwickelt wurde.

\section{Wo sind die Grenzen?}

Höhere Rationalität ist als Anspruch an Bewertungsmethoden zumindest irreführend, da ein wesentlicher Bestandteil Wertehaltungen und umweltpolitische Zielsetzungen sind, die subjektiv und normativ sind. Hinterfragen wir nun die beiden Bestandteile einer Ökobilanz im Hinblick auf deren Rationalität und Leistungsfähigkeit. Die Wirkungsabschätzung mit ihren naturwissenschaftlichen Bestandteilen beruht zum größeren Teil auf intersubjektiv überprüfbaren Verfahren, deren Charakter nicht so stark auf normativen Elementen beruht. Ihre Stärke liegt darin, daß sie als Vorbereitung zur eigentlichen Bewertung dient. Relevanter ist hier die Anforderung, die Informationsvielfalt entscheidungsunterstützend $\mathrm{zu}$ strukturieren und zu verdichten sowie dabei die naturwissenschaftlich begründbaren von den durch Wertehaltungen und umweltpolitische Zielsetzungen geprägten Bestandteilen zu differenzieren, um möglichst hohe Transparenz und Nachvollziehbarkeit zu gewährleisten. Diese Trennung leistet die Wirkungsabschätzung sicherlich. Einschränkend gilt es jedoch darauf hinzuweisen, daß in der nationalen wie internationalen Normung (nahezu identische) Listen bestehen, die die weitgehend akzeptierten ca. zehn Wirkungskategorien enthalten. Die Berechnung der Beiträge der verschiedenen Substanzen ist jedoch insbesondere international noch nicht abschließend geklärt: Bei ISO wurde erst ein Konsens darüber erzielt, daß die Kategorien „Treibhauseffekt" nach Maßgabe des IPCC in GWP 100-Einheiten (Global Warming Potential über den Zeitraum 100 Jahre) und „Ozonabbau" nach WMO (World Metereological Organisation) in ODP-Einheiten (Ozone Depletion Potential) berechnet und angegeben werden. Über die Berechnung der weiteren ca. acht Wirkungskategorien besteht noch kein Konsens.

Zusammenfassend betrachtet: Wir sehen die Wirkungsabschätzung als eine Hilfe zur Rationalisierung im Umgang mit Ökobilanzergebnissen an; sie erhöht deren Leistungsfähigkeit, da sie einen Beitrag zur Informationsverdichtung leistet; sie verschleiert nur begrenzt subjektive Werturteile und hilft damit sicherlich eine höhere Rationalität zu erreichen.

Gilt dies eben Gesagte aber auch für die zusammenfassende Beurteilung? Die problematische Frage ist demnach, wie der Prozeß der weiteren Beurteilung der Ergebnisse der Wirkungsabschätzung (und weiterer relevanter Teile der Sachbilanz) organisiert werden kann. Wer sind die Akteure, die unter Zuhilfenahme welchen Algorithmusses zu Empfehlungen oder Entscheidungen gelangen? Welche Hilfsmittel sind dabei „erlaubt"? Die bisherigen Vorgehensweisen sind 
sehr unterschiedlich, ein Trend zur Vereinheitlichung ist nicht erkennbar. Werfen wir also einen kurzen Blick in die Praxis:

- Einige Ökobilanzstudien gehen eher den verbal-deskriptiven Weg, indem sie auf Bewertungskriterien zurückgreifen, diese anwenden und argumentativ zu einem Gesamturteil verknüpfen.

- Andere Ökobilanzstudien verzichten auf eine explizite Bewertung und überlassen diese dem gesellschaftlichen Diskurs der interessierten Kreise.

- Eine Gewichtung mit Hilfe des Anlegens von Gewichtungsfaktoren nach dem Vorbild der Nutzwertanalyse ist eine weitere praktizierte Möglichkeit. Diese Gewichtungsfaktoren können auf definierten Bewertungskriterien oder auf rein subjektiven Wertmaßstäben beruhen.

- Die Verknüpfung der Wirkungsabschätzung und der Auswertung über einen bestimmten Algorithmus findet bei den Konzepten der „Stoffflußmethode" (vgl. BUS 1984) und der „Umweltbelastungspunkte“ (vgl. Braunschweig u.a 1993) statt. Hier wird also Bezug genommen auf einen - scheinbar - fachwissenschaftlich festgelegten Algorithmus.

Viele der derzeit eingesetzten Bewertungsmethodiken technisieren und „verschleiern“ den Vorgang der Bewertung: Der Bezug zu Wertungen und subjektiven Entscheidungen ist kaum mehr herstellbar. Eine tatsächliche Objektivität kann hierdurch nicht erzielt werden. Es gibt keine wertneutralen, nicht interessensgeleiteten (Be-) Wertungssysteme. Deshalb stellt sich die Aufgabe, Entscheidungsprozesse vorzustrukturieren und nicht $\mathrm{zu}$ ersetzen. Entscheidungen sind dann in Abwägung unterschiedlicher Aspekte und Interessen zu treffen. Eine „höhere“ Rationalität läßt sich nicht durch scheinbar einfache Ergebnisse erzielen. Vielmehr kommt es hierbei gerade auf den Akteursbezug und die prozeßhafte Einbettung des Bewertungsvorgangs in ein Netz pluraler Interessen an. Sollen Ökobilanzen in diesem Kontext nicht nur dazu dienen, einem kleinen Kreis von Personen mit (vermeintlich) homogen strukturierten Interessen zur Entscheidungsfindung zu dienen, so sollten weitere Personen partizipieren, wie beispielsweise eine unternehmensinterne Öffentlichkeit oder unternehmensexterne Kreise. Dies ist eine notwendige Voraussetzung zu ihrer Akzeptanz. Das bedeutet, daß Ökobilanzen unter bestimmten Rahmenbedingungen, die entweder für jede einzelne Studie definiert werden müssen, oder innerhalb eines institutionellen Zusammenhangs zu klären sind, Entscheidungsprozesse unterstïtzen und um eine Berücksichtigung der ökologischen Dimension „bereichern“ können.

\section{- Akteursbezug in Ökobilanzen}

Der fehlende Akteursbezug ist eine Schwachstelle der jüngsten Ökobilanzentwicklungen. Die Ökobilanzdiskussion wird weitgehend in Fachkreisen und damit auch auf dem entsprechenden Niveau gefuihrt. Die in der Norm formulierten Anforderungen sind hoch gesteckt und anspruchsvoll. Ungeklärt ist, ob Unternehmen unterhalb Konzerngröße über das entsprechende Know-how und die zeitlichen wie finanziellen Ressourcen verfügen und ob Verbraucher/innen über diese Kapazitäten verfügen. Das heißt, die Methodenentwicklung erfolgt unter weitgehender Ausblendung der Akteure und Zielgruppen. Betrachtet man die relevanten Akteure, so kommt man zu einer äußerst zwiespältigen Einschätzung der Leistungsfähigkeit der bisher entwickelten Bewertungsmethoden:

- Verbraucher/innen: Sie benötigen im Regelfall hochverdichtete Informationen, die in den Kaufentscheidungsprozeß einfließen. Hierzu muß also von anderen Akteuren eine Bewertung vorgenommen werden, die Verbraucher/innen nutzen können. Diese Bewertung muß verläßlich und stabil sein. Aus heutiger Sicht kommen hierfür nur Verfahren in Frage, die am Ende auf ein Ein-Punkt-Resultat oder einige wenige Aussagen hinauslaufen.

\section{- Betriebliche Entscheidungsträger: Hierbei} muß differenziert werden zwischen Fachabteilungen und der Geschäftsleitung. Letztere ist in der Regel gewöhnt, auf der Basis von möglichst wenigen, aber aussagekräftigen Informationen zu entscheiden. Hierfür müssen die Ökobilanzergebnisse hochverdichtet werden. Fachabteilungen, die diese hochverdichteten Ökobilanzaussagen betriebsintern erstellen, benötigen dazu sehr viel mehr Informationen, um die jeweilige Fragestellung der Ökobilanz handlungsrelevant beantworten zu können. Auf dieser Ebene eignen sich demnach Bewertungsverfahren, die mehrere, auf ihren technisch/ stofflichen Ausgangspunkt rückführbare Erkenntnisse liefern.

- Umweltpolitik: Produktempfehlungen der Umweltpolitik haben u. U. weitreichende Folgen und werden öffentlich intensiv diskutiert (und kritisiert). Die zugrundeliegenden Ökobilanzen müssen deshalb mit großer Sorgfalt erstellt wer- den und Vor- und Nachteile der verglichenen Produkte oder Produktsysteme ausführlich untersuchen. Die Produktempfehlungen müssen andererseits knapp, verständlich, eindeutig und allgemeingültig sein (siehe auch Anmerkungen bei Verbrauchern/innen). Solche Aussagen sind aus Ökobilanzen jedoch schwer abzuleiten, da deren Ergebnisse von den zugrundegelegten Annahmen und Systemgrenzen abhängig sind und damit keine Allgemeingültigkeit besitzen. Daneben verfolgt die Umweltpolitik bestimmte ökologische Ziele, die durch Ökobilanzergebnisse argumentativ untermauert werden sollen. Das für die Umweltpolitik geeignete Bewertungsverfahren sollte demnach eine Vielzahl an Informationen verarbeiten können, dabei Ausgewogenheit und Vollständigkeit gewährleisten, diese Informationen mit Umweltzielen in Einklang bringen und schließlich auch in knapper Form kommunizierbare Ergebnisse liefern. Als Grenze zeigt sich somit, daß Werturteile getroffen werden müssen, die verläßlich und zeitlich einigermaßen stabil sind und deren Zustandekommen transparent ist.

\section{Literafur}

- Ankele, K/, Rubik, F. (1997): Charakterisierung und Anwendungsmöglichkeiten ausgewählter Bewertungsverfahren. Berlin: IÖW-Schriftenreihe

- Braunschweig, A./ Förster, R./ Hofstetter, P./ MüllerWenk, R. (1993): Ökobilanzen für Unternehmungen. Eine Wegleitung für die Praxis. Bern u.a.

- BUS (Bundesamt für Umweltschutz) (1984): Ökobilanzen von Packstoffen. Bern

- Giegrich, J. u. a. (1995): Bilanzbewertung in produktbezogenen Ökobilanzen. Evaluation von Bewertungsmethoden, Perspektiven. Berlin: UBA-Texte 23/95

- Rubik, F./ Weskamp, C. (1996): Verbraucherschutz durch Produktkennzeichen. Berlin. IÖW-Schriftenreihe 95/96

- Rubik, F./ Teichert, V. (1997): Ökologische Produktpolitik. Stuttgart

- Schmitz, S. U. a. (1995): Ökobilanz von Getrönkeverpackungen. Berlin: UBA-Texte 52/95

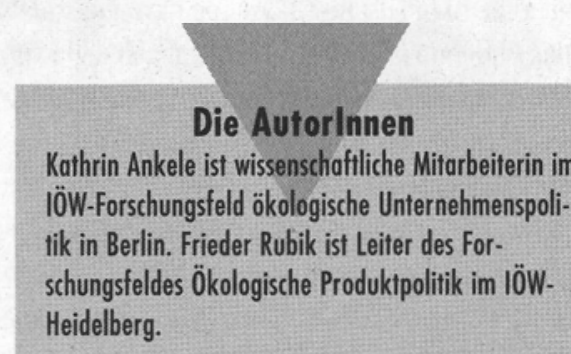

Kontakt: IÖW-Berlin: Tel. (030) 884594-0, I0̈WHeidelberg: Tel. (06221) 167954 
(c) 20I0 Authors; licensee IÖW and oekom verlag. This is an article distributed under the terms of the Creative Commons Attribution Non-Commercial No Derivates License (http://creativecommons.org/licenses/by-nc-nd/3.o/), which permits unrestricted use, distribution, and reproduction in any medium, provided the original work is properly cited. 